\title{
Noninvasive Detection of Coronary Artery Bypass Graft Patency by Intravenous Electron Beam Computed Tomographic Angiography
}

\author{
Shoji YAMAKAMI, ${ }^{1}$ MD, Junji TOYAMA, ${ }^{1}$ MD, Mitsuhiro OKAMOTO, ${ }^{1}$ MD, \\ Toyoaki Matsushita, ${ }^{1} \mathrm{MD}$, Yoshimasa MuraKAMI, ${ }^{1} \mathrm{MD}$, \\ Masaki OGATA, ${ }^{1} \mathrm{MD}$, Shigenori ITO, ${ }^{2} \mathrm{MD}$, Tatsuya FuKuTOMI, ${ }^{2} \mathrm{MD}$, \\ Naotsuka OKAYAMA, ${ }^{2} \mathrm{MD}$, and Makoto ITOH, ${ }^{2} \mathrm{MD}$
}

\begin{abstract}
SUMMARY
This study evaluates the usefulness of intravenous electron beam computed tomographic angiography (EBA) for the detection of coronary artery bypass graft patency in 43 patients ( 33 men and 10 women, mean age, 65 years) who had coronary artery bypass graft surgery. EBA was performed a few days before selective bypass graft angiography (SGA). Forty axial cross-sections of angiographic images of the heart were acquired consecutively by an electrocardiographic trigger signal at $40 \%$ of the RR interval, which corresponds to the end-systolic phase. EBA data were reconstructed as a three-dimensional shaded surface display of the heart and bypass grafts. Detectability of the patency of bypass grafts was evaluated, taking selective angiographic images of the bypass grafts as a gold standard. One hundred and nine grafts (96\%) out of 114 grafts were subjected to evaluation: 37 grafts were left internal mammary artery grafts (LIMA), 7 were right internal mammary artery grafts (RIMA), 6 were gastroepiploic artery grafts (GEA), 7 were free gastroepiploic artery grafts with venous drainage (free-GEA), 7 were radial artery grafts (RAG), and 45 were saphenous vein grafts (SVG). The sensitivity, specificity, positive predictive value, negative predictive value, and accuracy of EBA were $98 \%, 100 \%$, $100 \%, 91 \%$, and $98 \%$, respectively. EBA sampled at the end-systolic period was determined to be useful for the detection of coronary artery bypass graft patency and occlusion. (Jpn Heart J 2003; 44: 811-822)
\end{abstract}

Key words: Electron beam computed tomography, Coronary artery bypass grafting, Angiography

Postoperative angina occurs with a frequency of $30 \%$ in the first year after coronary artery bypass graft (CABG) surgery. ${ }^{1)}$ It may result from either graft occlusion, or progression of the atherosclerotic lesion of the native coronary

From the ${ }^{1}$ Division of Cardiology, Cardiovascular Center, Aichi Prefectural Owari Hospital, Ichinomiya, ${ }^{2}$ the Department of Internal Medicine and Bioregulation, Nagoya City University Medical School, Nagoya, Aichi, Japan.

Address for correspondence: Shoji Yamakami, MD, Division of Cardiology, Cardiovascular Center, Aichi Prefectural Owari Hospital, 2135, Kariyasuka, Yamato-cho, Ichinomiya, Aichi 491-0934, Japan.

Received for publication September 17, 2002.

Revised and accepted March 31, 2003. 
arteries. ${ }^{2)}$ Graft occlusion is reported to occur in $10-30 \%$ of grafts in the first 1-2 years, and in $45-55 \%$ by $10-12$ years after surgery. ${ }^{2-5)}$ Therefore, detection of graft patency or occlusion is principally important in diagnosing postoperative angina in detail as well as predicting the clinical course of patients. The gold standard for evaluating graft patency in $\mathrm{CABG}$ patients is selective bypass graft angiography (SGA). However, angiography is an invasive and costly procedure with some risks $^{6}$. . Therefore, noninvasive modalities such as exercise electrocardiography, ${ }^{7}$ exercise thallium scintigraphy, ${ }^{8)}$ exercise radionuclide angiography, ${ }^{9}{ }^{9}$ Doppler echocardiography, ${ }^{10)}$ and magnetic resonance imaging ${ }^{11-14)}$ have been developed, but they have been evaluated as having limited usefulness due to low diagnostic accuracy.

As an alternative to these procedures, electron beam computed tomography (EBCT) has been recently reported to be promising for the imaging of the coronary arteries because of its high spatial and temporal resolution and ability to trigger image acquisition with the use of electrocardiographic signals. A multicenter study using planar tomographic imaging with EBCT attained fairly good diagnostic accuracy (the sensitivity, specificity, and accuracy of detecting graft patency were $93.4 \%, 88.9 \%$, and $92.1 \%$, respectively). ${ }^{15)}$ For the purpose of obtaining more detailed information to understand the anatomy and status of graft patency, ${ }^{16-18)}$ recent advances in EBA technology have offered the challenge of constructing a three-dimensional image of the cardiac surface with coronary vessels. In this technology, a series of image data should be sampled at a specified trigger-time point of the cardiac cycle for several heart beats. However, it has not been established whether the time point should be chosen at the end-diastolic ${ }^{16,17)}$ or end-systolic phase. ${ }^{18)}$ EBA images scanned at the former phase of the cardiac cycle are apt to be distorted by movement of the coronary arteries associated with cardiac contraction. In this study, we adopted the latter phase by referring to the recent paper ${ }^{19)}$ by Mao, et al. We evaluated the diagnostic accuracy of EBA for determining the patency of 114 coronary artery bypass grafts including not only saphenous vein grafts (SVG) and left internal mammary artery grafts (LIMA), but also right internal mammary artery grafts (RIMA), gastroepiploic artery grafts (GEA), free gastroepiploic artery grafts with venous drainage (freeGEA), ${ }^{20)}$ and free radial artery grafts (RAG). We discuss the usefulness and limitations of this method.

\section{Methods}

The study population consisted of 43 patients who had undergone CABG surgery and subsequent SGA. The mean age ( \pm standard deviation) of the patients was $65 \pm 8$ years (range, 40 to 77 ); there were 33 men and 10 women. EBA was 
performed a few days before SGA. The average time from surgery to angiography was $2.6 \pm 0.7$ years (range, 1 month to 11 years).

There were a total of 114 grafts: 38 were LIMA, 8 were RIMA, 6 were GEA, 7 were free-GEA, 7 were RAG, and 48 were SVG. There were no composite grafts and no sequential grafts. Table I shows the types of bypass grafts and the sites of the bypass graft anastomoses. The patients had neither atrial fibrillation nor any contraindications for the administration of a contrast agent. Informed consent was obtained from each patient.

EBA: EBCT was obtained with an Imatron C-150 ultrafast computed tomographic scanner (Imatron, South San Francisco, California). The patients were positioned head first, scanned in the supine position, and shifted a few centimeters to the right side to adjust the field of view. To achieve vasodilation, sublingual nitroglycerin $(0.3 \mathrm{mg})$ was administered to all patients, who were then asked to hold their breath at near maximal lung capacity for each scan. To determine the position of the heart, five cross-sectional images of the chest (slice thickness, 3 $\mathrm{mm}, 7 \mathrm{~mm}$ gap between slices; field of view, $15 \mathrm{~cm}$; acquisition time, $100 \mathrm{~ms}$ ) were acquired with the scanner in the single-slice mode, and the position of the table was adjusted. For EBA, 40 axial cross-sectional images of the heart were acquired in the single-slice mode in $100 \mathrm{~ms}$ after an electrocardiographic trigger signal was generated at $40 \%$ of the RR interval (end-systolic phase). The matrix size was $512 \times 512$ and the field of view was $15 \mathrm{~cm}$ (pixel size, $0.29 \times 0.29 \mathrm{~mm}$ ). In-plane resolution was seven line pairs per centimeter. ${ }^{21)}$ The slice thickness was $3 \mathrm{~mm}$ with table increments of $2 \mathrm{~mm}$, to provide an overlap for optimal resolution of the bypass grafts and coronary arteries. Since the EBA range was limited to 8 $\mathrm{cm}$, it was difficult to detect both LIMA and GEA. Therefore, we detected not LIMA but GEA because the number of cases with GEA grafts was small. The contrast agent (Iopamidol, $370 \mathrm{mg}$ iodine $/ \mathrm{mL}$ ) was injected at a rate of $2 \mathrm{~mL} / \mathrm{sec}$. The rate of the contrast agent was increased to $4 \mathrm{~mL} / \mathrm{sec}$ for only 5 seconds after

Table I. Characteristics of Bypass Graft Anastomosis

\begin{tabular}{lccccccc}
\hline Grafts & LIMA & RIMA & GEA & free-GEA & RAG & SVG & Total \\
\hline Number & 38 & 8 & 6 & 7 & 7 & 48 & 114 \\
& & & & & & & \\
Anastomosis & 31 & 5 & 1 & 0 & 0 & 3 & 40 \\
$\quad$ to LAD & 0 & 1 & 5 & 6 & 3 & 17 & 32 \\
to RCA & 3 & 0 & 0 & 1 & 4 & 20 & 28 \\
to LCx & 3 & 2 & 0 & 0 & 0 & 8 & 13 \\
to diagonal branch & 1 & 0 & 0 & 0 & 0 & 0 & 1 \\
to high lateral branch & 1 & & & & & & \\
\hline
\end{tabular}


a delay of 23 seconds because the injected contrast agent was diluted by increased venous return in deep breathing. Imagings were commenced 30 seconds after the beginning of injection, and injection was stopped at the 20th image. The radiation dose was estimated to be less than $11 \mathrm{mSv}^{22,23)}$ The images were transferred to a workstation (Allegro, ISG Technologies, Toronto, Canada), and three-dimensional angiography was reconstructed. The number and course of bypass grafts were known to the investigators, but postprocessing was performed in a blinded fashion without knowledge of the angiography or the clinical condition of the patients. These data were rendered as a shaded surface display. As a general rule, the left appendage was excluded on an individual basis to allow better assessment of the proximal left coronary artery. Surgical clips were involved in the vessel lumens. Other structures (eg, pulmonary vessels and chest wall) were removed. An EBCT density of 80-100 Hounsfield units was chosen as the lower threshold so as to exclude the nonenhanced cardiac structure. Three-dimensional reconstructions were rendered interactively from at least six angles. The patency of each bypass graft was assessed from at least two angles, and patency or total occlusion was reported. Occlusion of bypass grafts was diagnosed if the grafts did not appear in the 3-dimensional reconstructions. In cases in which diagnosis by EBA was difficult, axial images of EBCT were used as a reference.

Coronary angiography and SGA: In all patients, coronary angiography and SGA were obtained in the standard fashion with more than 2 orthogonal views of each coronary artery and graft. Information about the number, location, and type of bypass graft (eg, saphenous, mammary, radial, native, or free gastroepiploic) was obtained from the surgical reports for all patients. SGA was analyzed using visual assessment by two experienced cardiologists unaware of the EBCT findings. Each bypass graft was assessed and reported as patent or total occlusion.

Statistical analysis: The ability of the EBA to correlate with angiographically confirmed bypass patency was evaluated by calculating the sensitivity, specificity, positive and negative predictive values, and accuracy using $2 \times 2$ contingency tables. Sensitivity was calculated as truly patent grafts/(truly patent grafts + falsely occluded grafts). Specificity was calculated as truly occluded grafts/(truly occluded grafts + falsely patent grafts). Positive predictive value was calculated as truly patent grafts/(truly patent grafts + falsely patent grafts). Negative predictive value was calculated as truly occluded grafts/(truly occluded grafts + falsely occluded grafts). Accuracy was calculated as (truly patent grafts + truly occluded grafts) / (truly patent grafts + falsely patent grafts + truly occluded grafts + falsely occluded grafts). The results of SGA served as a standard of reference. 
A

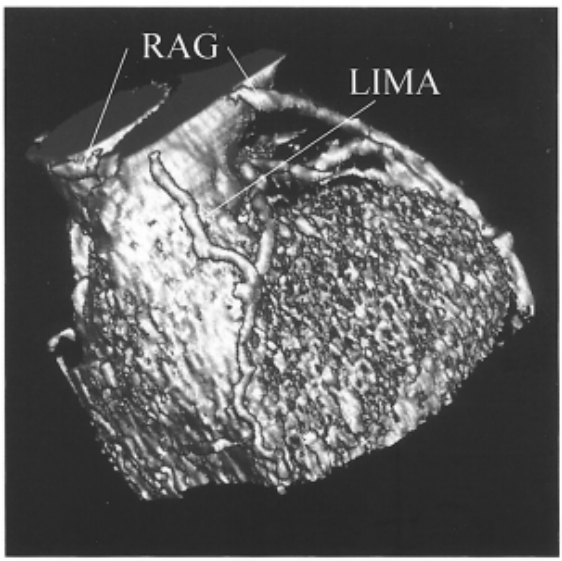

C

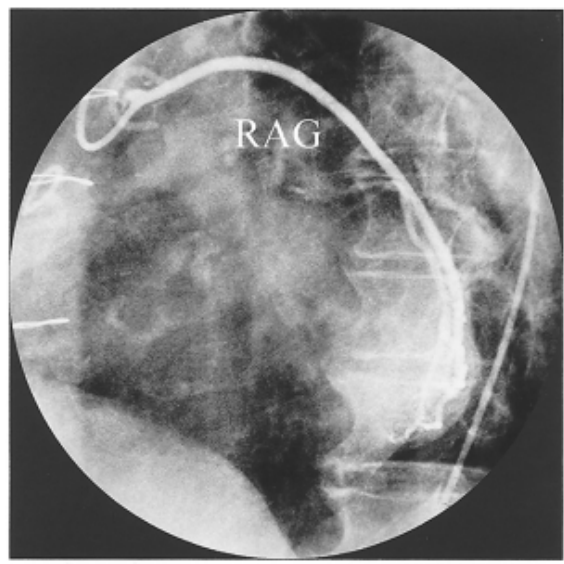

$\mathbf{E}$

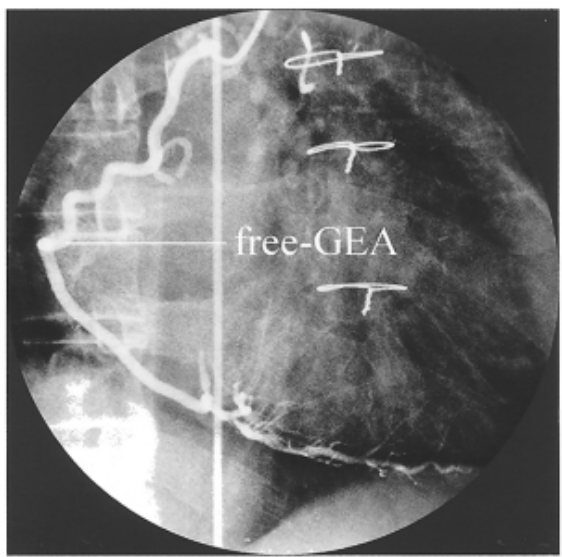

B

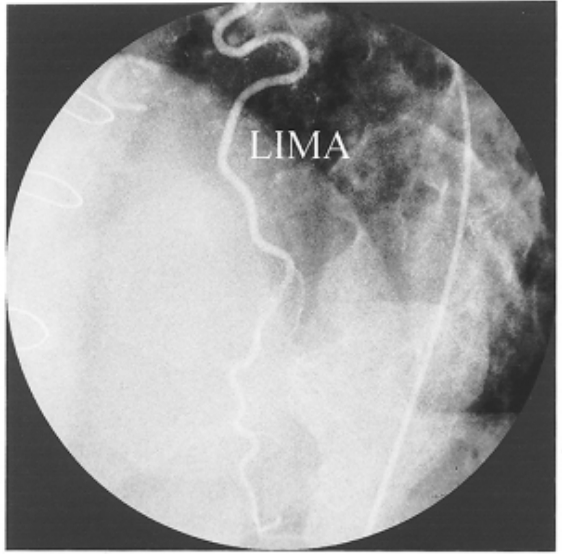

D

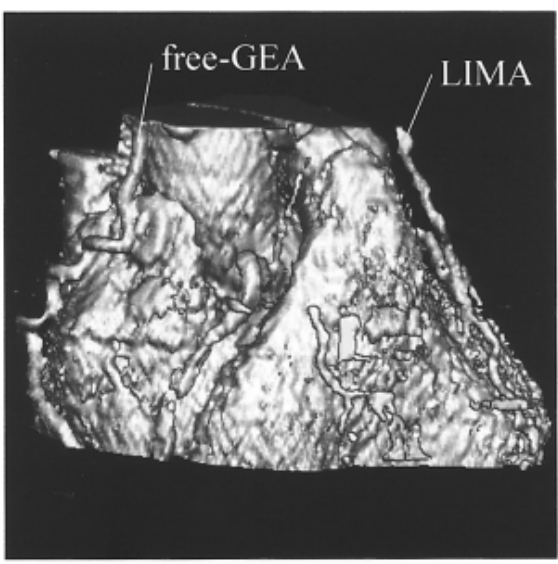

Figure 1. A 64-year-old male patient with a patent anastomosis LIMA to LAD, and a patent RAG to posterior lateral branch.

A: EBA image taken (left anterior oblique projection with cranial angulation) showing patency of LIMA to LAD and RAG to posterior lateral branch. B: SGA image in the left anterior oblique projection with cranial angulation showing a patent LIMA to LAD anastomosis. C: SGA image in the left anterior oblique projection showing a patent RAG to posterior lateral branch anastomosis. D: EBA image in the right anterior oblique projection showing a patent LIMA to LAD anastomosis, and a patent free-GEA to RCA. E: SGA image in the right anterior oblique projection showing a patent free-GEA to RCA anastomosis. 


\section{RESULTS}

EBCT examination was completed successfully without complications in all 43 patients. The scans were performed during a single breath-hold of 35 to 65 seconds in duration. With instruction, 38 patients $(88 \%)$ were able to hold their breath throughout the scan. A total of 106 to $136 \mathrm{~mL}$ of contrast medium was used per patient. Figure 1A shows an example of EBA which succeeded in the visualization of patency of a double bypass graft (LIMA to LAD, and RAG to posterior lateral branch) anastomosis. EBA visualization of the two bypass grafts was confirmed by SGA as shown in Figure 1B and Figure 1C. Figure 1D shows another EBA image aiming to visualize the patency of free-GEA with venous drainage to RCA. The EBA visualization is limited to a middle portion of the graft in comparison with that of SGA (Figure 1E). The EBA image does not cover the complete length of the graft due to the limited spatial coverage. Figure 2 is an example of an unsuccessful EBA image of GEA to RCA anastomosis, in which a stenotic legion was visualized as indicated. The flow of the GEA graft to RCA, appeared to be satisfactory based on the density of contrast medium along the graft, however, the existence of stenosis (arrow in Figure 2A) could not be excluded from the graft image. On the other hand, this bypass graft was proved to be patent by SGA alone (Figure 2B). Figure 3A shows a case with occluded LIMA to LAD and patent SVG to diagonal branch anastomoses were proved by SGA (Figure 3B, 3C). EBA did not visualize the LIMA, and also did not detect the diagonal branch because this branch was too small in diameter to be visualized by EBA (Figure 3A).
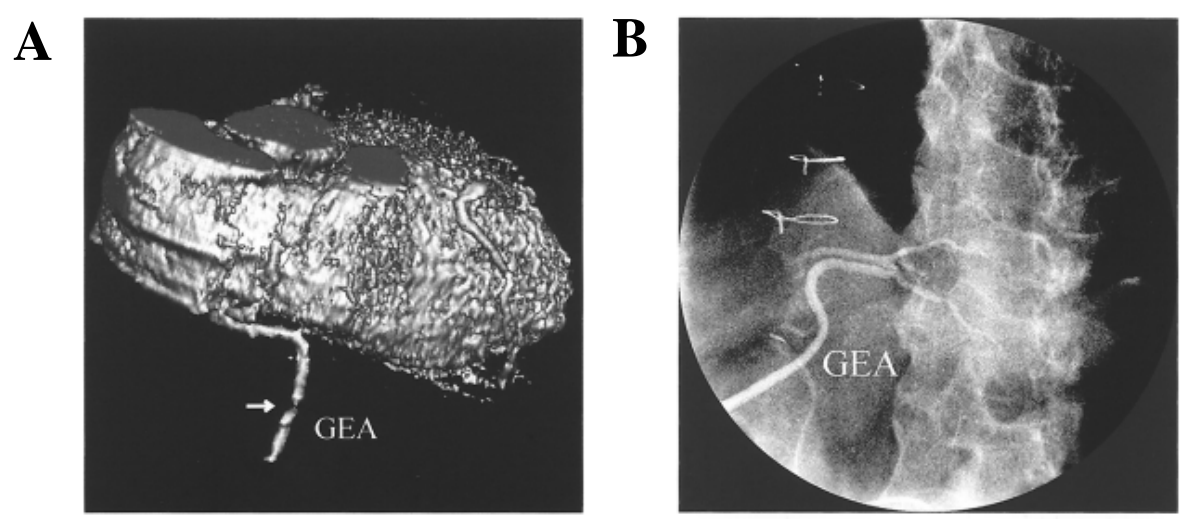

Figure 2. A 55-year-old man with a patent GEA to RCA. A: EBA image in the right anterior oblique projection with cranial angulation visualizing a patent, but highly stenotic GEA (arrow) to RCA anastomosis. By referring to the axial images, we recognized that the stenotic image was produced by the patient's motion. B: SGA image in the left anterior oblique projection with cranial angulation visualizing a fully patent GEA to RCA anastomosis. 
A

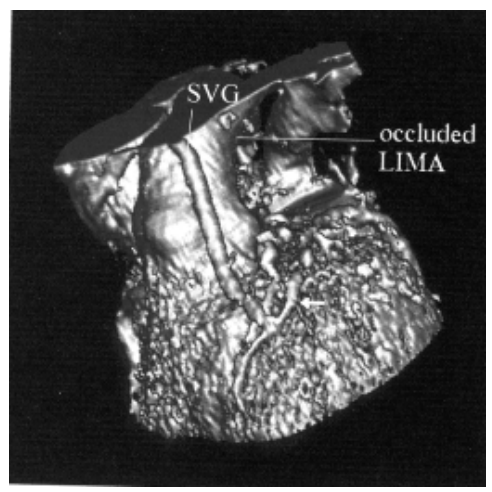

C

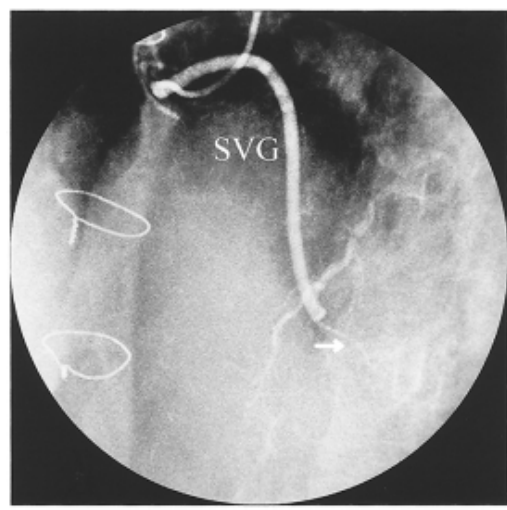

B

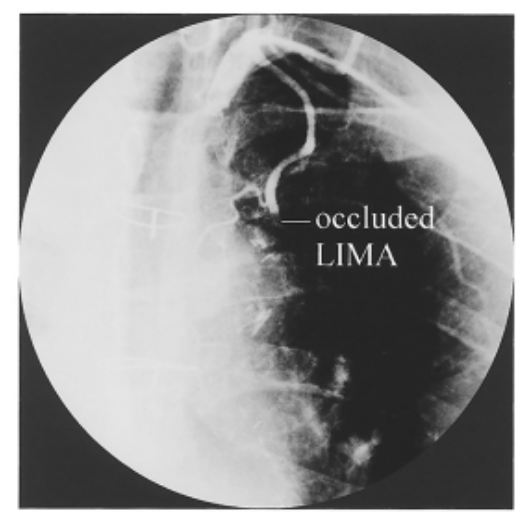

Figure 3. A 70-year-old man with an occluded LIMA to LAD and a patent SVG to diagonal branch. A: EBA image taken in the left anterior oblique projection with cranial angulation showing an occluded LIMA to LAD anastomosis, and a patent SVG to diagonal branch anastomosis. Great cardiac vein is indicated by arrow. The diagonal branch is not shown because of the narrow diameter. B: SGA image in the right anterior oblique projection showing an occluded LIMA. C: SGA image in the left anterior oblique projection with cranial angulation showing a patent SVG to diagonal branch anastomosis. Diagonal branch is indicated by arrow.

Overall accuracy for bypass graft patency: We excluded 5 grafts from evaluation because their images were out of the range of 40 EBCT frames. A total of 109 grafts (37 LIMA, 7 RIMA, 6 GEA, 7 free-GEA, 7 RAG, and 45 SVG) were evaluated for occlusion or patency. All 45 vein grafts (100\%) and 62 of 64 arterial grafts $(97 \%)$ were successfully visualized on EBA images. The overall rate of success in EBA visualization of the patent bypass grafts attained $98 \%$.

Diagnostic accuracy of patency or occlusion in 109 grafts was calculated (Table II). Bypass graft angiography revealed 89 patent grafts, 87 of which were identified as patent on EBA. One LIMA to LAD was incorrectly judged as occluded by EBA because of its narrow diameter measured as $1.1 \mathrm{~mm}$ by quantitative coronary angiography (Figures 4A, B, C). One RAG to LCx was incorrectly judged as occluded by EBA due to poor image quality, probably because the patient was physically well built. As for the 10 grafts which were diagnosed as occluded by SGA, EBA succeeded in diagnosing all of the cases as occluded. 
Table II Comparison of SGA Diagnosis and EBA Visualization of Graft Patency or Occlusion

\begin{tabular}{lcccccc}
\hline & SGA diagnosis & \multicolumn{2}{c}{ EBA visualization } & \multicolumn{2}{c}{ SGA diagnosis } & \multicolumn{2}{c}{ EBA visualization } \\
\hline grafts & patent & yes & no $(\%)^{*}$ & occluded & yes & no $(\%)^{*}$ \\
LIMA & 29 & 28 & $1(97)$ & 8 & 8 & $0(100)$ \\
RIMA & 6 & 6 & $0(100)$ & 1 & 1 & $0(-)$ \\
GEA & 5 & 5 & $0(100)$ & 1 & 1 & $0(-)$ \\
free-GEA & & 7 & $0(100)$ & & 0 & $0(-)$ \\
RAG & 7 & 6 & $1(83)$ & 0 & 0 & $0(-)$ \\
SVG & 35 & 35 & $0(100)$ & 10 & 10 & $0(100)$ \\
\end{tabular}

* percentage of successful EBA visualization of graft patency or occlusion proved by SGA.
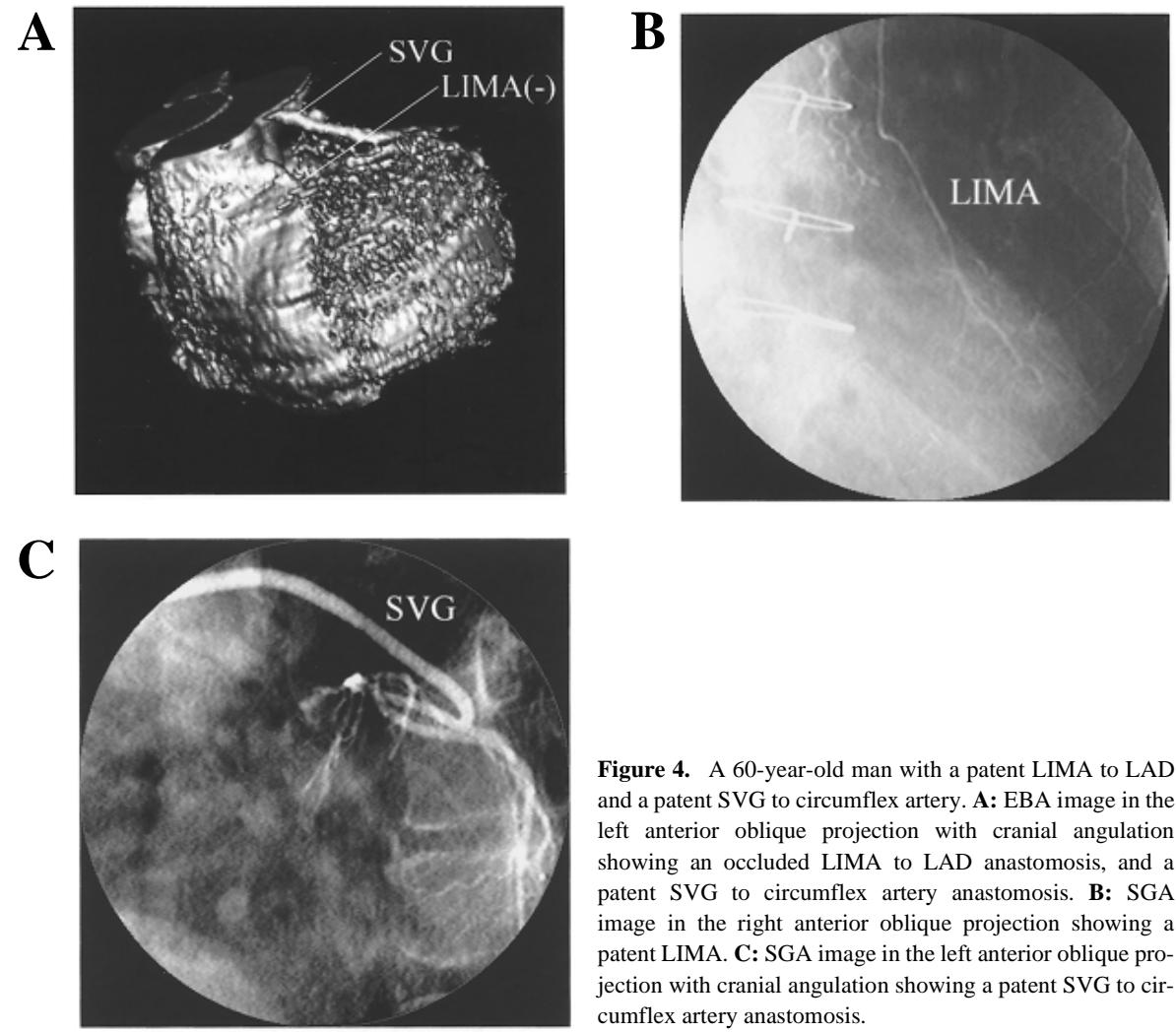

Figure 4. A 60-year-old man with a patent LIMA to LAD and a patent SVG to circumflex artery. A: EBA image in the left anterior oblique projection with cranial angulation showing an occluded LIMA to LAD anastomosis, and a patent SVG to circumflex artery anastomosis. B: SGA image in the right anterior oblique projection showing a patent LIMA. C: SGA image in the left anterior oblique projection with cranial angulation showing a patent SVG to circumflex artery anastomosis.

Table III shows the sensitivity, specificity, positive predictive value, negative predictive value, and diagnostic accuracy of EBA in diagnosing patent and occluded bypass grafts, dividing them into arterial and vein grafts groups. The sensitivity and specificity were 96 and $100 \%$ in the former group, thus providing 
Table III. Diagnostic Accuracy of Electron Beam Computed Tomographic Angiography (EBA) on Patent and Occluded Coronary Bypass Grafts

\begin{tabular}{lccc}
\hline & $\begin{array}{c}\text { Arterial grafts } \\
(n=64)\end{array}$ & $\begin{array}{c}\text { SVG } \\
(n=45)\end{array}$ & $\begin{array}{c}\text { all } \\
(n=109)\end{array}$ \\
\hline Sensitivity (\%) & 96 & 100 & 98 \\
Specificity (\%) & 100 & 100 & 100 \\
Positive predictive value (\%) & 100 & 100 & 100 \\
Negative predictive value (\%) & 83 & 100 & 91 \\
Accuracy (\%) & 97 & 100 & 98 \\
\hline
\end{tabular}

a positive predictive value, negative predictive value, and accuracy of $100 \%$, $83 \%$, and $97 \%$, respectively, whereas all the values were $100 \%$ in the latter group.

Thus, the overall sensitivity, specificity, positive predictive value, negative predictive value, and accuracy were $98 \%, 100 \%, 100 \%, 91 \%$, and $98 \%$, respectively.

\section{DISCUSSION}

Although the patency of coronary artery grafts can be assessed by several noninvasive methods, ${ }^{7-14,23)}$ it has been generally accepted that EBCT is one of the best ways to detect graft patency. However, in the early stage of these studies, EBCT was only used as a tool for time-density curves of bypass grafts rather than to produce two- or three-dimensional images of the lumen of a coronary artery bypass graft. ${ }^{15,24,25)}$

Achenbach, et al showed that the assessment of bypass graft patency using three-dimensional images (EBA) obtained with EBCT yielded an accuracy of $100 \%$ in 25 patients with 56 bypass grafts. ${ }^{16)} \mathrm{Ha}$, et al found the sensitivity and specificity of EBA in revealing LIMA patency were $80 \%$ and $82.4 \%$, and the sensitivity and specificity of EBA in revealing SVG patency were $91.7 \%$ and $91.1 \%$, respectively. ${ }^{17)}$ They attributed the difference in accuracy to the fact that LIMA grafts are smaller in diameter and more likely to have a significant number of surgical clips, which can produce beam-hardening and stellate artifacts that obscure the images. Their EBA was scanned at a trigger point of $80 \%$ of the RR interval. Scanning with such a delay in the diastolic period aimed to minimize the motion of the ventricles. But the atria are contracting at that time point, causing motion artifacts in the right coronary artery and left circumflex arteries. ${ }^{26)}$ Therefore, Mao, et al recommend a 40-50\% R-R interval as an electrocardiographic trigger time to minimize cardiac artery motion. ${ }^{19)}$ Hoshi, et $a l^{18)}$ reported that EBA triggered at $45 \%$ of the RR interval succeeded in diagnosing 42 of 44 arterial grafts and all 47 venous grafts as patent (sensitivity: 95\%, 100\%), and all 7 venous 
grafts as occlusive (specificity: 100\%). As a result, the overall sensitivity and specificity were calculated to be $98 \%$ and $88 \%$, respectively. Our study triggered at $40 \%$ of the RR interval showed good diagnostic accuracy of EBA in revealing LIMA and SVG patency; the sensitivity and specificity of EBA in revealing LIMA patency were $97 \%$ and $100 \%$, and the sensitivity and specificity of EBA in revealing SVG patency were $100 \%$ and $100 \%$, respectively.

For better graft patency over the long term, there has been routine use of multiple arterial grafts for $\mathrm{CABG}^{27)}$ As in situ arterial grafts, GEA is secondary to the internal mammary artery. ${ }^{28)}$ Moreover, RAG $^{29)}$ and free-GEA ${ }^{20)}$ have been applied clinically. We investigated 43 patients with 114 coronary artery bypass grafts including LIMA, RIMA, GEA, RAG, and free-GEA by EBA. Although 5 of the 114 bypass grafts had to be excluded from evaluation, graft patency and occlusion were diagnosed with $98 \%$ accuracy.

Limitations: EBA and our method have several limitations. The current EBCT scanner model has limited spatial coverage during a single breath-hold and during the first pass of the contrast agent. Our EBA protocol called for a slice thickness of $3 \mathrm{~mm}$ with table increments of $2 \mathrm{~mm}$ following previous reports of intravenous CT coronary angiography ${ }^{30-32)}$ for the detection of stenoses. But we could not evaluate 5 grafts because the EBA range was limited to $8 \mathrm{~cm}$. Achenbach, et al used a slice thickness of $3 \mathrm{~mm}$ with table increments of $3 \mathrm{~mm}$, and the scan area resulted in a $12 \mathrm{~cm}$ slab. ${ }^{16)}$ Their protocol may be better than ours from the standpoint of scan area rather than actual detection of stenoses. Other limitations include motion artifacts caused by cardiac motion, respiratory motion, and poor electrocardiographic gating. In our protocol, however, motion artifacts might be fewer than in other studies because scans were triggered at $40 \%$ of the RR interval (end-systolic phase) in comparison to their scans that were triggered at $80 \%$ (end-diastolic phase). It is desirable that EBA is performed in patients with sinus rhythm for reduction of motion artifacts. Moreover, a partial volume effect, exposure to ionizing radiation, potential reactions to contrast agent, and the need for cannulation of the vein also could be disadvantageous for some patients.

There were four bypass grafts with angiographically high-grade stenoses; three grafts were LIMA to LAD, and one was RIMA to LAD. The former three grafts with angiographically high-grade stenoses were diagnosed as patent without high-grade stenosis by EBA. These errors may be attributed to image interference caused by reflection from the metallic vessel clips. Since larger numbers of metallic vessel clips are attached to internal mammary artery grafts than other grafts, such interference may occur more easily in LIMA and RIMA grafts. But the error could be prevented using volume rendering as a postprocessing technique. 
The latest generation of multislice spiral computed tomography scanners can reconstruct images with a data acquisition window of $250 \mathrm{~ms} .{ }^{33)}$ Achenbach, et al reported that multislice spiral computed tomography with retrospectively ECG gated image reconstruction permits coronary artery stenoses but its clinical use may presently be limited due to degraded image quality in a substantial number of cases, mainly due to rapid coronary motion. ${ }^{34)}$ Multislice spiral computed tomographic angiography will be applicable for the detection of patency of CABG.

In conclusion, EBA triggered at $40 \%$ of the RR interval is clinically useful for noninvasive direct detection of coronary artery bypass graft patency because of its high accuracy.

\section{ACKNOWLEDGMENTS}

We are indebted to the technical staff of the Cardiovascular Center of Aichi Prefectural Owari Hospital for their invaluable assistance, especially Hideki Satsurai and Manabu Yoshimoto.

\section{REFERENCES}

1. CASS principal investigators and their associates: Coronary Artery Surgery Study (CASS): a randomized trial of coronary artery bypass surgery-quality of life in patients randomly assigned to treatment groups. Circulation 1983; 68: 951-60.

2. Campeau L, Enjalbert M, Lesperance J, et al. Atherosclerosis and late closure of aortocoronary saphenous vein grafts: sequential angiographic studies at two weeks, one year, five to seven years, and ten to twelve years after surgery. Circulation 1983; II-1-7.

3. Loop FD. Progress in surgical treatment of coronary atherosclerosis (part I). Chest 1983; 84: 611-22.

4. Lawrie GM, Morris GC Jr, Chapman DW, et al. Patterns of patency of 596 grafts up to seven years after aortocoronary bypass. J Thorac Cardiovasc Surg 1977; 73: 443-8.

5. Fitzgibbon GM, Kafka HP, Leach AJ, et al. Coronary bypass graft fate and patient outcome: angiographic follow-up of 5065 grafts related to survival and reoperation in 1388 patients during 25 years. J Am Coll Cardiol 1996; $28:$ 616-26.

6. Wyman RM, Safian RD, Portway V, et al. Current complications of diagnostic and therapeutic cardiac catheterization. J Am Coll Cardiol 1988; 12: 1400-6.

7. Fox K, Selwyn A, Jonathan A, et al. Electrocardiographic mapping after exercise for evaluation of coronary artery bypass graft surgery. Am J Cardiol 1979; 44: 1251-6.

8. Pfisterer M, Emmenegger H, Schmitt HE, et al. Accuracy of serial myocardial perfusion scintigraphy with thallium-201 for prediction of graft patency early and late after coronary artery bypass surgery: a controlled prospective study. Circulation 1982; 66: 1017-24.

9. Higginbotham MB, Belkin RN, Morris KG, et al. Value and limitation of biplane rest and exercise radionuclide angiography for assessing individual bypass grafts: a prospective study. J Am Coll Cardiol 1986; 7: 1004-14.

10. Diebold B, Theroux P, Bourassa MG, et al. Noninvasive assessment of aortocoronary bypass graft patency using pulsed Doppler echocardiography. Am J Cardiol 1979; 43: 10-6.

11. Gomes AS, Lois JF, Drinkwater DC, et al. Coronary artery bypass grafts: visualization with MR imaging. Radiology 1987; 162: 175-9. 
12. Aurigemma GP, Reichek N, Axel L, et al. Noninvasive determination of coronary artery bypass graft patency by cine magnetic resonance imaging. Circulation 1989; 80: 1595-602.

13. Vrachliotis TG, Bis KG, Aliabadi D, et al. Contrast-enhanced breath-hold MR angiography for evaluating patency of coronary artery bypass grafts. AJR 1997; 168: 1073-80.

14. White RD, Caputo GR, Mark AS, et al. Coronary artery bypass graft patency: noninvasive evaluation with MR imaging. Radiology 1987; 164: 681-6.

15. Stanford W, Brundage BH, McMillan R, et al. Sensitivity and specificity of assessing coronary bypass graft patency with ultrafast computed tomography: results of a multicenter study. J Am Coll Cardiol 1988; 12: 1-7.

16. Achenbach S, Moshage W, Ropers D, et al. Noninvasive, three-dimensional visualization of coronary artery bypass grafts by electron beam tomography. Am J Cardiol 1997; 79: 856-61.

17. Ha JW, Cho SY, Shim WH, et al. Noninvasive evaluation of coronary artery bypass graft patency using threedimensional angiography obtained with contrast-enhanced electron beam CT. AJR 1999; 172: 1055-9.

18. Hoshi T, Yamauchi T, Kanauchi T, et al. Three-dimensional computed tomography angiography of coronary artery bypass graft with electron beam tomography. J Cardiol 2001; 38: 197-202 (in Japanese).

19. Mao S, Lu B, Oudiz RJ, et al. Coronary artery motion in electron beam tomography. J Comput Assist Tomogr 2000; 24: 253-8.

20. Matsuura A, Yasuura K, Yoshida K, et al. Transplantation of the en bloc vascular system for coronary revascularization. J Thorac Cardiovasc Surg 2001; 121: 520-5.

21. Achenbach S, Moshage W, Ropers D, et al. Value of electron-beam computed tomography for the noninvasive detection of high-grade coronary-artery stenoses and occlusions. N Engl J Med 1998; 339: 1964-71.

22. Agatston AS, Janowitz WR, Hildner FJ, et al. Quantification of coronary artery calcium using ultrafast computed tomography. J Am Coll Cardiol 1990; 15: 827-32.

23. Stanford W, Thompson BH, Weiss RM. Coronary artery calcification: clinical significance and current methods of detection. AJR 1993; 161: 1139-46.

24. Bateman TM, Gray RJ, Whiting JS, et al. Cine computed tomographic evaluation of aortocoronary bypass graft patency. J Am Coll Cardiol 1986; 8: 693-8.

25. Stanford W, Krachmer M, Galvin JR. Ultrafast computed tomography in assessing coronary bypass grafts. Am J Cardiac Imaging 1991; 5: 21-8.

26. Kaufmann RB, Sheedy PF, Breen JF, et al. Interobserver and intraobserver reliability for scoring quantifications. Radiology 1994; 190: 347-52.

27. Bergsma TM, Grandjean JG, Voors AA, et al. Low recurrence of angina pectoris after coronary artery graft with bilateral internal thoracic and right gastroepiploic arteries. Circulation 1998; 97; 2402-5.

28. Suma H, Fukumoto H, Takeuchi A. Coronary artery bypass grafting by utilizing in situ right gastroepiploic artery: basic study and clinical application. Ann Thorac Surg 1987; 44: 394-7.

29. Acar C, Jebara VA, Portoghese M, et al. Revival of the radial artery for coronary artery grafting. Ann Thorac Surg 1992; 54: 652-60.

30. Rensing BJ, Bongaerts A, van Geuns RJ, et al. Intravenous coronary angiography by electron beam computed tomography: a clinical evaluation. Circulation 1998; 98: 2509-12.

31. Schmermund A, Rensing BJ, Sheedy PF, et al. Intravenous electron-beam computed tomographic coronary angiography for segmental analysis of coronary artery stenoses. J Am Coll Cardiol 1998; 31: 1547-54.

32. Nakanishi T, Ito K, Imazu M, et al. Evaluation of coronary artery stenoses using electron-beam CT and multiplaner reformation. J Comput Assist Tomogr 1997; 21: 121-7.

33. Ohnesorge B, Flohr T, Becker C, et al. Cardiac imaging by means of electrocardiographically gated multisection spiral CT: Initial experience. Radiology 2000; 217: 564-71.

34. Achenbach S, Giesler T, Ropers D, et al. Detection of coronary artery stenoses by contrast-enhanced, retrospectively electrocardiographically-gated, multislice spiral computed tomography: Circulation 2002; 103: 2535-8. 